\title{
Late Reinfection With a Different SARS-CoV-2 Clade in a Patient With Refractory Arterial Hypertension: a Case Report.
}

\section{Antonio Galiana}

Hospital General Universitario de Elche Javier García-Abellán

Hospital General Universitario de Elche

Marta Fernández-González

Hospital General Universitario de Elche

Nieves Gonzalo-Jiménez

Hospital General Universitario de Elche

Montserrat Ruiz-García

Hospital General Universitario de Elche

María Andreo

Hospital General Universitario de Elche

\section{Selene Falcón}

Hospital General Universitario de Elche

\section{Mar Masiá}

Hospital General Universitario de Elche

Félix Gutiérrez ( $\nabla$ gutierrez_fel@gva.es )

Universidad Miguel Hernandez de Elche https://orcid.org/0000-0002-9485-6867

\section{Case Report}

Keywords: SARS-CoV-2, reinfection, COVID-19, antibody response, case report

Posted Date: April 8th, 2021

DOI: https://doi.org/10.21203/rs.3.rs-392287/v1

License: (c) (i) This work is licensed under a Creative Commons Attribution 4.0 International License. Read Full License 


\section{Abstract}

Background: Differentiating between persistent infection with intermittent viral shedding and reinfection with SARS-CoV-2 remains challenging. Although a small number of cases with genomic evidence of second infection have been reported, limited information exists on frequency and determinants of reinfection, time between infections, and duration of immunity after the primary infection.

Case presentation: We report a reinfection with SARS-CoV-2 in a 52-year old male whose primary infection was diagnosed in May 2020, during the first wave of the pandemic in Spain, and the second occurred eight months later, in January 2021. We present a complete data set including results from realtime polymerase chain reaction, serology and genome sequencing confirming reinfection with a different clade. Noteworthy was that the patient was immunocompetent but had multiple cardiometabolic comorbidities, including refractory arterial hypertension, that might increase the individual risk in COVID19.

Conclusions: This case of reinfection with SARS CoV-2 occurring several months after the primary infection reports the longest time interval between reinfection and initial infection described to date. It raises concerns on the duration of protective immunity, suggesting that it may begin to wane in patients who acquired the initial infection during the first wave of the pandemic. The potential contributing role of arterial hypertension and cardiometabolic comorbidities as risk factors for reinfection deserves investigation.

\section{Background}

A year after the COVID-19 pandemic began, uncertainty remains on the strength and duration of immunity after first SARS-CoV-2 infection. Although only a small number of cases with genomic evidence of second infection have been reported thus far [1-7], recent data suggest that protective immunity may indeed be short-lived [8], thus raising concerns about the risk of reinfections as the pandemic progresses and immunity begins to wane.

Unfortunately, diagnosing reinfections remains challenging, requiring banking samples from first infection and performing genomic sequencing to distinguish reinfection from persistent viral shedding. As a result, limited data exist on frequency and determinants of SARS-CoV-2 reinfection. Therefore, studying every SARS-CoV-2 reinfection is important to describe the natural history of COVID-19 and to gain insight into the factors that may increase susceptibility to secondary infections.

Most reinfections described in the literature have occurred from three to five months after the initial episode and most patients had antibodies against SARS-CoV-2 at the time of reinfection [7]. We have recently confirmed a second infection with a different clade of SARS-CoV-2 in a patient with multiple cardiometabolic risk factors for COVID-19 whose initial infection occurred eight months before, in the first wave of the pandemic in Spain. The patient did not have anti-SARS-CoV-2 antibodies before onset of reinfection. 


\section{Case Presentation}

A 52 year-old male with severe arterial hypertension was admitted to hospital on May 10, 2020, due to uncontrolled blood pressure with acute kidney failure. The patient did not have fever nor respiratory symptoms. He had a past medical history of refractory hypertension with hypertensive heart disease and chronic kidney disease, morbid obesity, dyslipidemia and sleep apnea-hypopnea syndrome. He was receiving therapy with five antihypertensive agents of different classes, including angiotensin receptor blockers. Real-time polymerase chain reaction (PCR) screening for SARS-CoV-2 performed on a nasopharyngeal swab (NPS) before hospital admission was positive (cycle threshold value $[\mathrm{Ct}]=30$ ). The chest radiograph did not show any abnormal findings and all the laboratory biomarkers, including neutrophil:Iymphocyte ratio, serum levels of C-reactive protein, interleukin 6, D-dimer and ferritin were within the normal range. After controlling blood pressure, he had an uneventful recovery and was discharged home five days after hospital admission. The patient did not receive corticosteroids, other immunomodulators, or antivirals.

He was contacted by phone one month after discharge and offered follow-up at the outpatient clinic to repeat serologic tests and NPS for SARS-CoV-2 at two and six months after hospital admission.

Unfortunately, he missed the two-month appointment but came to the clinic for the six-month visit, which was performed on December 14,2020. At that date, the PCR on NPS was negative and specific immunoglobulin $\mathrm{G}(\mathrm{IgG})$ against the SARS-CoV-2 internal nucleocapsid protein (N-IgG) and surface S1 domain of the spike protein (S-IgG) (Anti-SARS-CoV-2 IgG ELISA, Euroimmun, Lubeck, Germany) were not present.

On January 11, 2021, 240 days after the first infection, he presented to hospital with a 3 days history of fever, myalgia and asthenia. The chest radiograph was normal and the oxygen saturation was $95 \%$. SARS-CoV-2 infection was confirmed by PCR testing on NPS with a Ct value of 18 . He had a serum creatinine level of $1.8 \mathrm{mg} / \mathrm{dL}$ (normal value, $0.66-1.25 \mathrm{mg} / \mathrm{dL}$ ) and a mild elevation of liver transaminases. The rest of the laboratory results were normal as with the initial infection. He did not have SARS-CoV-2-specific IgG antibodies (anti-SARS-CoV-2 S-IgG and N-IgG, Virclia Vircell, Granada, Spain and anti-SARS-CoV-2 S1/S2-IgG, Liaison DiaSorin, Saluggia, Italy). Specific IgM (anti-SARS-CoV-2 IgM, Liaison DiaSorin) and IgM + IgA (Virclia Vircell) were also negative. An extensive immunological investigation including lymphocyte subpopulations in peripheral blood ruled out immunodeficiency. He received symptomatic care and oral dexamethasone with resolution of fever and myalgia, and was discharged on January 13,2021 . On February 15,2021 , the PCR on NPS was negative. At that time, specific S-IgG, N-lgG and $\lg M+\lg A$ (Virclia Vircell) and S1/S2-IgG (Liaison DiaSorin) were present.

\section{Genome sequencing}

Genome sequencing of the viral genome was performed on stored aliquots of the NPS samples collected on May 10, 2020 (sample A) and January 11, 2021 (sample B) following the ARTIC SARS-CoV-2 amplicon sequencing protocol for MinION version V3 (https://www.protocols.io/view/ncov-2019-sequencing- 
protocol-bbmuik6w). The sequencing protocol uses $400 \mathrm{bps}$ amplicons in a tiled fashion across the whole SARS-COV2 genome. After samples library synthesis, the samples were sequenced with the MinION nanopore sequencer (Oxford Nanopore Technologies, Oxford, UK) using the Ligation Sequencing kit with the Native Barcoding Expansion. The sequencing runs were performed using the flow cell FLOMN106D and extended until both samples got 50.000 high quality reads. Sequencing base calling was made using the nanopore guppy basecaller.

Downstream analysis was performed following the ARTIC nCoV-2019 bioinformatics protocol (https://artic.network/ncov-2019/ncov2019-bioinformatics-sop.html), a protocol which takes the output from the sequencing protocol to get the consensus genome SARS-CoV-2 sequences. The protocol follows the base calling step and make a de-multiplexing sample sequences followed by a sampling mapping step polishing and a sequence consensus generation.

Phylogenetic analysis of both specimens was done using web server Nextstrain (https://nextstrain.org/), with the SARS-CoV-2 database Nextclade (https://clades.nextstrain.org/) to identify the clade, mutation calling and phylogenetic placement of the SARS-COV2 genomes.

Single Nucleotide Variants (SNVs) analysis against GISAID reference genomes showed differences between the two samples. Sample A was member of the clade 20A EU1 as showed their hallmark SNVs T445C, A23403G, C28932T. Sample B was member of the clade 20B as their hallmark SNVs were T10836C, A23403G, G28883C. Both samples shared some SNVs as G6167C, C14408T, A23122T and A268016G. SNVs are shown on Table 1 and mapped on the SARS-CoV-2 genome in Fig. 1. Phylogenetic analysis of both samples against GISAID genomes database using Nextclade web server analysis are shown in Fig. 1. 
Table 1

Single Nucleotide Variants analysis versus reference genome, and aminoacid substitutions in the two samples.

\begin{tabular}{|c|c|c|c|}
\hline \multirow[t]{2}{*}{ Nucleotide mutations versus reference genome } & \multicolumn{2}{|c|}{ Sample* } & \multirow[t]{2}{*}{ Aminoacid substitutions } \\
\hline & A & B & \\
\hline T445C & Yes & No & - \\
\hline G6167C & Yes & Yes & ORF1a: V1968L \\
\hline T10836C & No & Yes & ORF1a: V3524A \\
\hline C14408T & Yes & Yes & ORF1b: P314L \\
\hline T16213C & Yes & No & ORF1b: Y916H \\
\hline T22669C & Yes & No & - \\
\hline A23122T & Yes & Yes & - \\
\hline A23403G & Yes & Yes & S: D614G \\
\hline A25900G & Yes & No & ORF3a: T170A \\
\hline A268016G & Yes & Yes & - \\
\hline C27944T & Yes & No & - \\
\hline G28883C & No & Yes & $\mathrm{N}: \mathrm{G} 204 \mathrm{R}$ \\
\hline C28932T & Yes & No & $\mathrm{N}: \mathrm{A} 220 \mathrm{~V}$ \\
\hline G29645T & Yes & No & - \\
\hline
\end{tabular}

\section{Discussion And Conclusions}

This case presentation adds to the still limited knowledge on second infections with SARS-CoV-2. Here, a complete data set including results from PCR, serology and genome sequencing is provided allowing us to confirm reinfection with a different clade eight months after an asymptomatic initial infection. The first infection was with the $20 \mathrm{~A}$ clade and the reinfection with the 20B. The two clades are characterized by a D614G substitution in the $S$ gene, and reflect the most frequent circulating strains in Spain at that time [9]. The underlying cardiometabolic comorbidities in this case, in particular the presence of severe refractory hypertension, gives rise to doubts to whether this condition, one of the most common preexisting comorbidities in patients with COVID-19 [10], could eventually increase the individual risk of SARS-CoV-2 reinfection.

The interval between reinfection and initial infection was the longest reported so far in the literature and probably reflects waning immunity overtime. Recent studies have emphasized rapid decay of anti-SARS- 
CoV-2 antibodies with large portions of a study population seroreverting within a few months [8]. Indeed, in this case report, we could document that anti-SARS-CoV-2 antibodies were not present shortly before onset of the second infection and at the time of presentation, thus highlighting the role of humoral immunity in the protection against reinfections.

This case of reinfection in an immunocompetent host several months after an asymptomatic infection raises concerns on the duration of protective immunity and suggest that it may begin to wane in patients who acquired the initial infection during the first wave of the pandemic. The potential contributing role of arterial hypertension and cardiometabolic comorbidities as risk factors for reinfection deserves investigation.

\section{Abbreviations}

SARS-CoV-2: Severe Acute Respiratory Syndrome Coronavirus 2; COVID-19: Corona Virus Disease 19; PCR: Polymerase Chain Reaction; NPS: Naso Pharyngeal Swab; IgG: Immunoglobulin G; SNV: Single nucleotide variant;

\section{Declarations}

\section{Ethics approval and consent to participate}

This study was performed in line with the principles of the Declaration of Helsinki. Approval was granted by the Institutional Review Board of Hospital Universitario de Elche (PI46/2020). Informed consent was obtained from the patient for publication of this case report.

\section{Consent for publication}

We have obtained consent for publication from the patient.

\section{Availability of data and materials}

Genoma sequencing results are available from the corresponding author on reasonable request.

\section{Competing Interest}

On behalf of all authors, the corresponding author states that there is no conflict of interest.

\section{Funding}

FG, MM and MF were supported by RD16/0025/0038 project as a part of the Plan Nacional Research + Development + Innovation $(\mathrm{R}+\mathrm{D}+\mathrm{I})$ and cofinanced by Instituto de Salud Carlos III - Subdirección General de Evaluación y Fondo Europeo de Desarrollo Regional; Instituto de Salud Carlos III (Fondo de Investigaciones Sanitarias [grant number PI16/01740; PI18/01861; CM19/00160; COV20/00005]). JGA was supported by CM20/00066. AG was supported by COV20/00156. The funding agencies had no role 
in the design and conduct of the study; collection, management, analysis, and interpretation of the data; preparation, review, or approval of the manuscript; or decision to submit the manuscript for publication.

\section{Authors' contributions}

AG and FG conceived the paper and wrote the first draft; MM, JGA, SF, MA and MFG participated in data acquisition; AG, NG and MR performed genoma sequencing. All authors critically revised the manuscript, and all authors approved the submission.

\section{Acknowledgements}

This report would not have been possible without the collaboration of all the medical, nursing and laboratory staff involved in the COVID-19 Healthcare Program and the COVID-19 Care Teams of Hospital General Universitario de Elche.

\section{References}

1. To KK-W, Hung IF-N, Ip JD, Chu AW-H, Chan W-M, Tam AR, et al. Coronavirus Disease 2019 (COVID19) Re-infection by a phylogenetically distinct severe acute respiratory syndrome coronavirus 2 strain confirmed by whole genome sequencing. Clin Infect Dis. 2020. https://doi.org/10.1093/cid/ciaa1275.

2. Tillett RL, Sevinsky JR, Hartley PD, Kerwin H, Crawford N, Gorzalski A, et al. Genomic evidence for reinfection with SARS-CoV-2: a case study. Lancet Infect Dis. 2021;21:52-8.

3. Mulder M, van der Vegt DSJ, Oude Munnink BB, GeurtsvanKessel CH, van de Bovenkamp J, Sikkema RS, et al. Reinfection of SARS-CoV-2 in an immunocompromised patient: a case report. Clin Infect Dis. 2020. https://doi.org/10.1093/cid/ciaa1538.

4. Larson D, Brodniak SL, Voegtly LJ, Cer RZ, Glang LA, Malagon FJ, et al. A case of early re-infection with SARS-CoV-2. Clin Infect Dis. 2020. https://doi.org/10.1093/cid/ciaa1436.

5. Colson P, Finaud M, Levy N, Lagier JC, Raoult D. Evidence of SARS-CoV-2 re-infection with a different genotype. J Infect. 2020. https://doi.org/10.1016/j.jinf.2020.11.011.

6. Elslande JV, Vermeersch P, Vandervoort K, Wawina-Bokalanga T, Vanmechelen B, Wollants E, et al. Symptomatic SARS-CoV-2 reinfection by a phylogenetically distinct strain. Clin Infect Dis. 2020. https://doi.org/10.1093/cid/ciaa1330.

7. Babiker A, Marvil C, Waggoner JJ, Collins M, Piantadosi A. The importance and challenges of identifying SARS-CoV-2 reinfections. J Clin Microbiol. 2020. https://doi.org/10.1128/JCM.02769-20.

8. Ibarrondo FJ, Fulcher JA, Goodman-Meza D, Elliott J, Hofmann C, Hausner MA, et al. Rapid decay of anti-SARS-CoV-2 antibodies in persons with mild Covid-19. N Engl J Med. 2020;383:1085-7.

9. Díez-Fuertes F, Iglesias-Caballero M, García-Pérez J, Monzón S, Jiménez P, Varona S, et al. A Founder Effect Led Early SARS-CoV-2 Transmission in Spain. J Virol. 2021;95:e01583-20. 
10. Svensson P, Hofmann R, Häbel H, Jernberg T, Nordberg P. Association between cardiometabolic disease and severe COVID-19: a nationwide case-control study of patients requiring invasive mechanical ventilation. BMJ Open. 2021;11:e044486.

\section{Figures}

Sample A

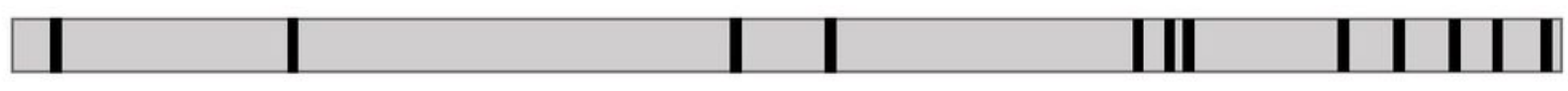

Sample B

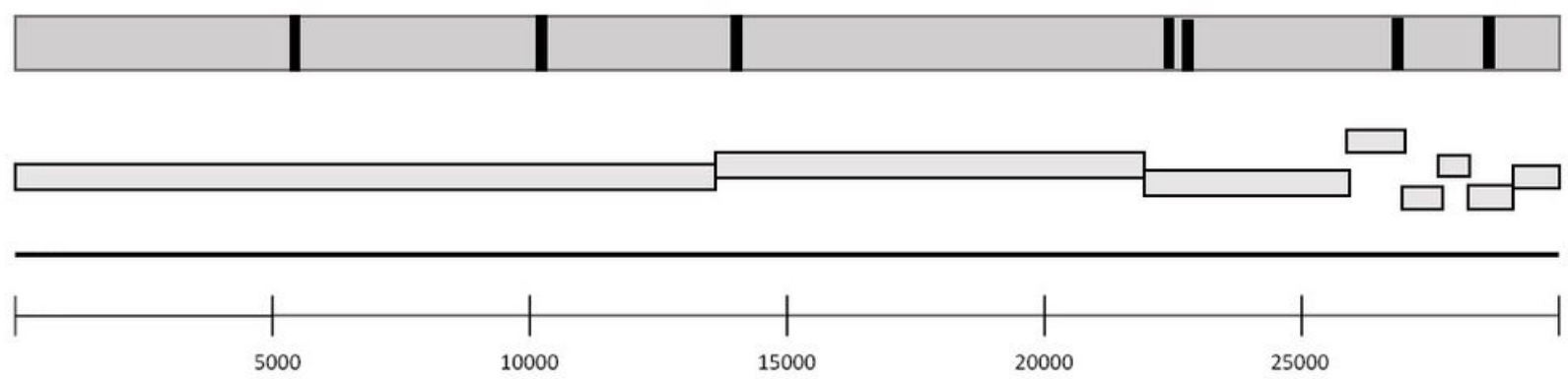

Figure 1

Single Nucleotide Variants in the two NPS samples mapped on the SARS-CoV-2 genome. Sample A was obtained on May 10, 2020 and sample B on January 11, 2021. 